\title{
Pyramid of Malpighi
}

National Cancer Institute

\section{Source}

National Cancer Institute. Pyramid of Malpighi. NCI Thesaurus. Code C33436.

Red-colored, striated conical structures of the medullary portion of the kidney. The number of these structures varies from eight to eighteen. 